Conclusion ACNES is an uncommon cause of abdominal pain in the pregnant patient.

The fibrous ring in the rectus muscle, through which the medial cutaneous nerve travels, causes nerve ischemia when compressed. Risk factors include abdominal distension, subcutaneous oedema, and previous surgery especially pfannelstein incision. The syndrome, commonly affecting the $7^{\text {th }}-12^{\text {th }}$ intercostal nerves, causes unilateral pain, hypoesthesia/hyperesthesia and a positive Carnett's sign. Management with local anaesthetic injection relieves symptoms and confirms the diagnosis.

This diagnosis should be considered in cases of severe abdominal pain when investigations are normal. The advantages of early diagnosis are relief of maternal pain and avoidance of delivering a preterm fetus.

\section{PM.74 PLACENTAL ABRUPTION SECONDARY TO ACUTE PANCREATITIS CAUSED BY HYPERTRIGLYCERIDAEMIA}

doi:10.1136/archdischild-2013-303966.155

WHJ Ince, AC McKelvey, J Harrad, J-W Chan. Norfolk and Norwich University Hospital, Norwich, UK

Introduction Placental abruption secondary to pancreatitis is rare. There are two cases in the literature. There are no previous reported cases of placental abruption secondary to pancreatitis caused by hypertriglyceridaemia.

Case We present the case of a 30 year old low risk primagravida who presented at 39 weeks and 6 days gestation with severe epigastric pain. Her bedside observations revealed tachypnoea but were otherwise normal. Urinalysis revealed proteinuria $(++)$. Vaginal examination was consistent with early labour. Initial differential diagnosis included a surgical emergency, pre-eclampsia, labour and placental abruption. Initial CTG was normal but became pathological and she went on to have emergency caesarean section within two hours of arrival. Operative findings included milky white ascitic fluid on opening the abdomen and a retroplacental clot. Chemical pathology telephoned to inform the team that her blood tests appeared lipaemic and she had an amylase of 1043 U/L (20-120 $\mathrm{U} / \mathrm{L}$ ) and triglycerides of $134 \mathrm{mmol} / \mathrm{L}(<1.70 \mathrm{mmol} / \mathrm{L})$. She was transferred to intensive care and has had a complicated recovery, which included cardiac arrest on her first postoperative day. At the time of writing, she remains on intensive care (her seventh week) and is being considered for a pancreatectomy for severe haemorrhagic pancreatitis.

Conclusion Acute pancreatitis should be considered in all patients presenting with upper abdominal pain. It can be difficult to diagnose and present non-specifically, particularly in the latter stages of pregnancy. It is an important cause of abruption. Triglycerides increase in pregnancy and are a well established cause of pancreatitis.

\section{PM.75 SAFETY OF FONDAPARINUX IN PREGNANCY- EXPERIENCE OF A NORTH WEST TERTIARY REFERRAL CLINIC}

doi:10.1136/archdischild-2013-303966.156

\section{N Musial, L Byrd. Saint Mary's Hospital, Manchester, UK}

Venous thromboembolism (VTE) is amongst the leading causes of maternal death in developed countries. Several series have confirmed the safety and efficacy of LMWHs in pregnancy and it has become the favoured anticoagulant. Adverse skin reactions to LMWHs are rare but recognised events, whereupon a particular LMWH may be successfully replaced with another one. However if the skin symptoms do not improve an alternative must be sought.

Fondaparinux is a synthetic pentasaccharide. Whilst it has been extensively studied for use both in surgical prophylaxis and treatment of thromboembolic diseases; its use in pregnancy is less well documented.

We report 4 pregnancies in 3 women using Fondaparinux which adds to the available literature. All required thromboprophylaxis because of previous pregnancy associated VTE when they demonstrated broad cross-reactivity between several heparins and/or heparinoids.

In 3 pregnancies Fondaparinux was commenced in the first trimester and continued until 6 weeks postpartum. All continued without event resulting in vaginal delivery of well grown babies at term. There was no minor or major maternal bleeding (mean blood loss $250 \mathrm{mls}$ ) or thromboembolic event reported during the pregnancy or post-partum period. All babies were breastfed without effect. There was no congenital abnormality or neonatal bleeding. In a $4^{\text {th }}$ pregnancy LMWH was initially deferred until 20 weeks gestation where upon recurrent allergic skin reaction led to the change to Fondaparinux. Review at 37 weeks gestation was preempted by a complaint of reduced fetal movements with pathological CTG necessitating emergency caesarean section at her base hospital.

\section{PM.76 A QUESTION OF PROBITY: PHANTOM BLOOD GLUCOSE VALUE RECORDING IN A SELF-MONITORING PREGNANT DIABETIC WOMAN}

doi:10.1136/archdischild-2013-303966.157

A Adeyemo, M Hicks, A Kulkarni, Z Ramsey-Marcelle. North Middlesex Hospital, London, UK

Introduction A successful doctor-patient relationship is based on perceived mutual trust. The importance of this is underscored in pregnant diabetic women where self monitoring of blood glucose (SMBG) levels by patients' informs and directs shared clinical decisions which impact significantly on their lifestyle, diet and medication adjustments so that a good pregnancy outcome is achieved.

Case Report A 29 yr old para2 woman with type 2 diabetes was booked and followed up in the combined antenatal diabetic clinic through her third pregnancy. She required metformin and subcutaneous insulin injections to control her blood glucose prior to conception. These were continued in pregnancy with an increase in the frequency of blood glucose monitoring. At 28 weeks of pregnancy, it was noted that blood glucose values recorded in her logbook over the course of 8 weeks consistently showed fasting levels $<5 \mathrm{mmol} / \mathrm{l}$ and $1 \mathrm{hr}$ postprandial levels $<7 \mathrm{mmol} / \mathrm{l}$ despite $\mathrm{HbA}_{1} \mathrm{C}$ levels of $72 \mathrm{mmols} / \mathrm{mol}(8.7 \%)$ and ultrasound scan showing fetal macrosomia and significant polyhydramnios. A review of her glucometer identified difference in the values stored in the memory and that recorded in her logbook. This discrepancy was brought to her notice and she divulged falsifying the values. She was delivered by emergency caesarean section for fetal compromise at 32 weeks gestation.

Conclusion Accuracy of home metres and diary logs needs to be confirmed at regular intervals, and SMBG values should not be the only criterion for diabetes management during pregnancy.

\section{PM.77 SUSPECT THE UNEXPECTED!: A RARE CASE OF UTERINE NECROSIS AND ASYMPTOMATIC RUPTURE FOLLOWING INDUCED MISCARRIAGE AT 20 WEEKS GESTATION}

doi:10.1136/archdischild-2013-303966.158

T Thomas, N Mufti, M Allam. Wishaw General Hospital, Wishaw, UK

A 38 year-old parous lady with an unscarred uterus was admitted for induction of medical miscarriage following intrauterine death of 